\title{
Juvenile hyaline fibromatosis: a rare oral disease case report and literature review
}

\author{
Liang $\mathrm{Xia}^{1 \#}$, Yuhua $\mathrm{Hu}^{2 \#}$, Chunye Zhang ${ }^{2}$, Dandan $\mathrm{Wu}^{1}$, Yang Chen ${ }^{1}$ \\ ${ }^{1}$ Department of Oral and Cranio-maxillofacial Surgery, The Ninth People's Hospital, Shanghai Jiao Tong University School of Medicine, National \\ Center of Stomatology, National Clinical Research Center for Oral Diseases, Shanghai Key Laboratory of Stomatology, Chinese Academy of \\ Medical Sciences Oral and Maxillofacial Regenerative Medicine Innovation Unit, Shanghai, China; ${ }^{2}$ Department of Oral Pathology, The Ninth \\ People's Hospital, Shanghai Jiao Tong University School of Medicine, National Center of Stomatology, National Clinical Research Center for Oral \\ Diseases, Shanghai Key Laboratory of Stomatology, Chinese Academy of Medical Sciences Oral and Maxillofacial Regenerative Medicine Innovation \\ Unit, Shanghai, China \\ \#These authors contributed equally to this work and share the co-first author. \\ Correspondence to: Yang Chen; Dandan Wu. No. 639, Zhizaoju Road, Huangpu District, Shanghai 200011, China. Email: whitesurgeon@163.com; \\ 179554314@qq.com.
}

\begin{abstract}
Juvenile hyaline fibromatosis (JHF) is a rare recessive autosomal hereditary disorder characterized by papulonodular skin, gingival hyperplasia, flexural joint contractures, and osteolytic bone lesions. Worldwide, less than 70 cases have been reported. JHF is thought to be a disorder of collagen metabolism which is caused by genomic sequence variations in the ANTXR2/CMG2 gene and is characterized by homogenous amorphous hyaline material and fibrous tissue. JHF is most commonly diagnosed in infants and in children less than 5 years. We report a 28-month-old child of a consanguineous marriage who presented with severe gingival hyperplasia, multiple facial nodules, posterior occipital tumors, joint contractures, and osteolytic bone lesions. His limbs and fingers cannot be straightened, with a posture of frog pose. The occlusal and incisal surfaces of the teeth were completely covered with the gingival overgrowth. The gingival hypertrophy and facial swellings were surgically removed, with pathological features of monomorphic spindled cell proliferation surrounded by an abundant amorphous hyaline matrix. The genome sequencing was performed that a homozygous nucleotide mutation of ANTXR2/CMG2 gene was found. We outline this particular patient's presentation, followed by a discussion highlighting the characteristics that change with the condition and the treatments of this disease. The treatment of JHF is generally symptomatic treatment and requires multidisciplinary care. Physical rehabilitation has been advocated for a lifetime.
\end{abstract}

Keywords: Juvenile hyaline fibromatosis (JHF); ANTXR2; gingival hypertrophy; surgical excision; case report

Submitted Apr 15, 2021. Accepted for publication Jul 22, 2021.

doi: $10.21037 / \mathrm{tp}-21-169$

View this article at: https://dx.doi.org/10.21037/tp-21-169

\section{Introduction}

Juvenile hyaline fibromatosis (JHF) is a rare and progressive autosomal recessive connective tissue disorder (1). Less than $70 \mathrm{JHF}$ cases have been reported worldwide (2). JHF is most commonly diagnosed in young infants and in children younger than 5 years, although there are a few reports of new diagnosis in adult life (3), which is characterized by multiple subcutaneous nodular lesions, gingival hypertrophy, joint contractures, and osteolytic lesions on bones, but normal intelligence (4). JHF may be associated with movement limitations, feeding problems, malnutrition, and recurrent infections. JHF can be progressive and the manifestations tend to be obvious as age increases. There is no gender or ethnic predilection. One-third of affected children are siblings and some have been born to consanguineous parents (5). In addition to the clinical features, confirmation of diagnosis requires 

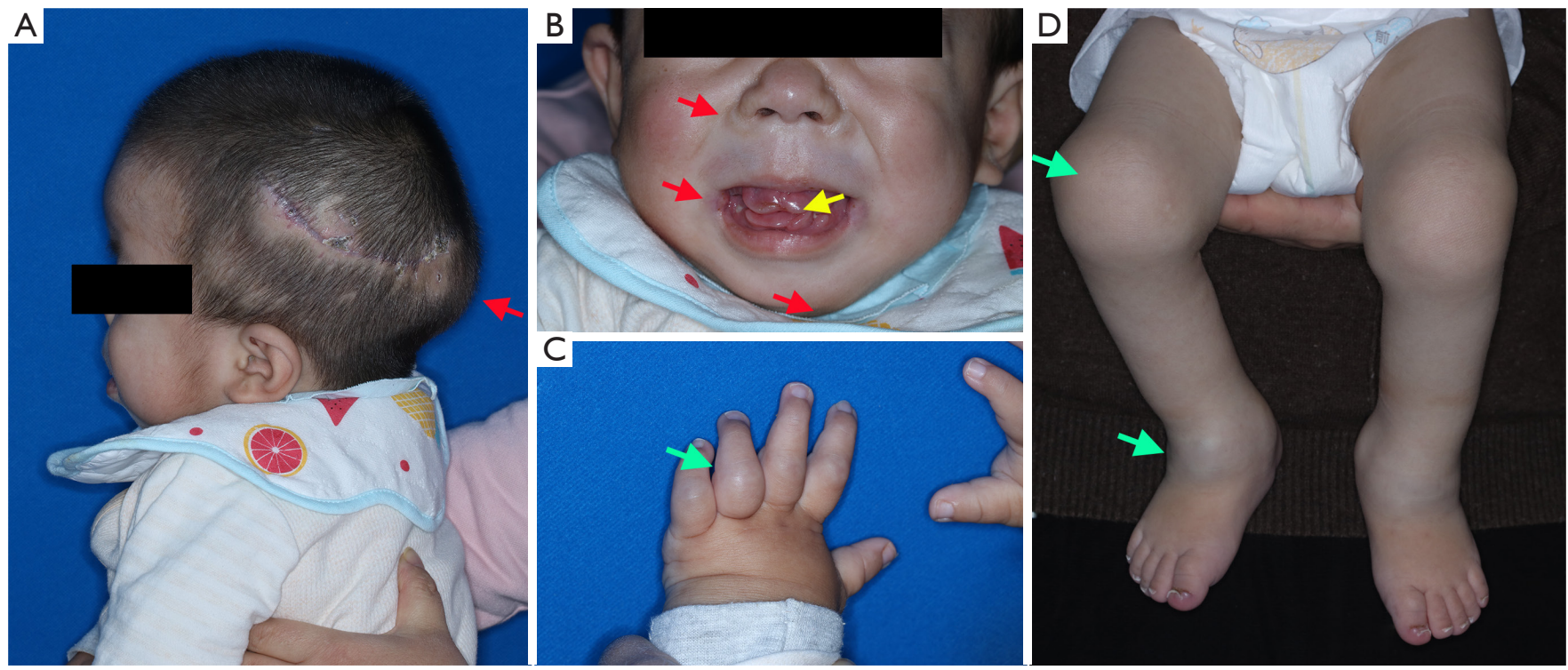

Figure 1 Patient with juvenile hyaline fibromatosis. (A) Occipital scar after operation with relapsed tumor (red arrow); (B) multiple masses under the nasal alar on both sides of the oral cavity and submandibular region (red arrows). Gingiva hyperplasia almost covers the incisors (yellow arrow); (C) contracture of the finger joint with dyskinesia (green arrow); (D) tumor formation and deformity of the knee and ankle joint, unable to straighten (green arrows). These images are published with the consent from the patient's parent or legal guardian.

microscopic examination of nodule biopsy specimens, which typically shows spindle-shaped fibrous cells with an abundant number of broadly hyalinized matrices (1). Herein we present a child patient diagnosed with JHF. We present the following case in accordance with the CARE reporting checklist (available at https://dx.doi.org/10.21037/tp-21169).

\section{Case presentation}

A 28-month-old boy of a consanguineous marriage (3rd generation) was brought to our department, with the chief complaint of swollen gums, inability to eat and masses on face since he was 1 year old. History revealed that he was born with swellings on the occipital scalp, and underwent scalp tumor resection when he was less than 1 year old. After the surgery, his parents found his gingiva was slowly overgrowing, disabling feeding and mastication. Subsequently, subcutaneous swellings and nodules appeared on his face. Upper limbs, lower limbs and fingers cannot be straightened, with a posture of frog pose.

He was $10.8 \mathrm{~kg}$ on admission with growth retardation. Examination revealed that his skin was harder and thicker than usual. Postoperative scars and recurrent tumors were seen on the occipital region (Figure 1A). Diffuse subcutaneous nodules were seen occupying the subnasal region, bilateral corner of the mouth, and submental area (Figure 1B). Joint contractures were evident at the elbows, knees, and fingers of the hands and toes with flexion deformity at these joints (Figure 1C,1D). Swelling was evident at the elbows and knee joints (Figure 1D). The mobility of the fingers of the hands, the toes of the feet and the joints in general was restricted and limited.

Intraoral examination revealed a diffuse swelling of the maxillary and mandibular gingivae covering the teeth almost to the incisal surfaces (Figure 1B). Cranio-maxillofacial computed tomography (CT) revealed multiple diffuse irregular soft tissue thickening with unclear boundaries and multiple bone destruction with low-density shadows (Figure $2 A-2 H$ ).

The gingival hyperplasia and subcutaneous facial nodules were surgically removed (Figure $3 A$ ). Grossly, the nodules were of grayish-white, and the cut surface showed a solid fibroid gray-white appearance (Figure 3B). The histopathological slides showed there were poorly circumscribed lesions in the submucosa composed of a sparse population of uniform spindle cells embedded in an abundant homogenous eosinophilic hyaline matrix, without a discernable mitotic phase (Figure 4A-4C). Immunohistochemical studies showed that the spindle-shaped 

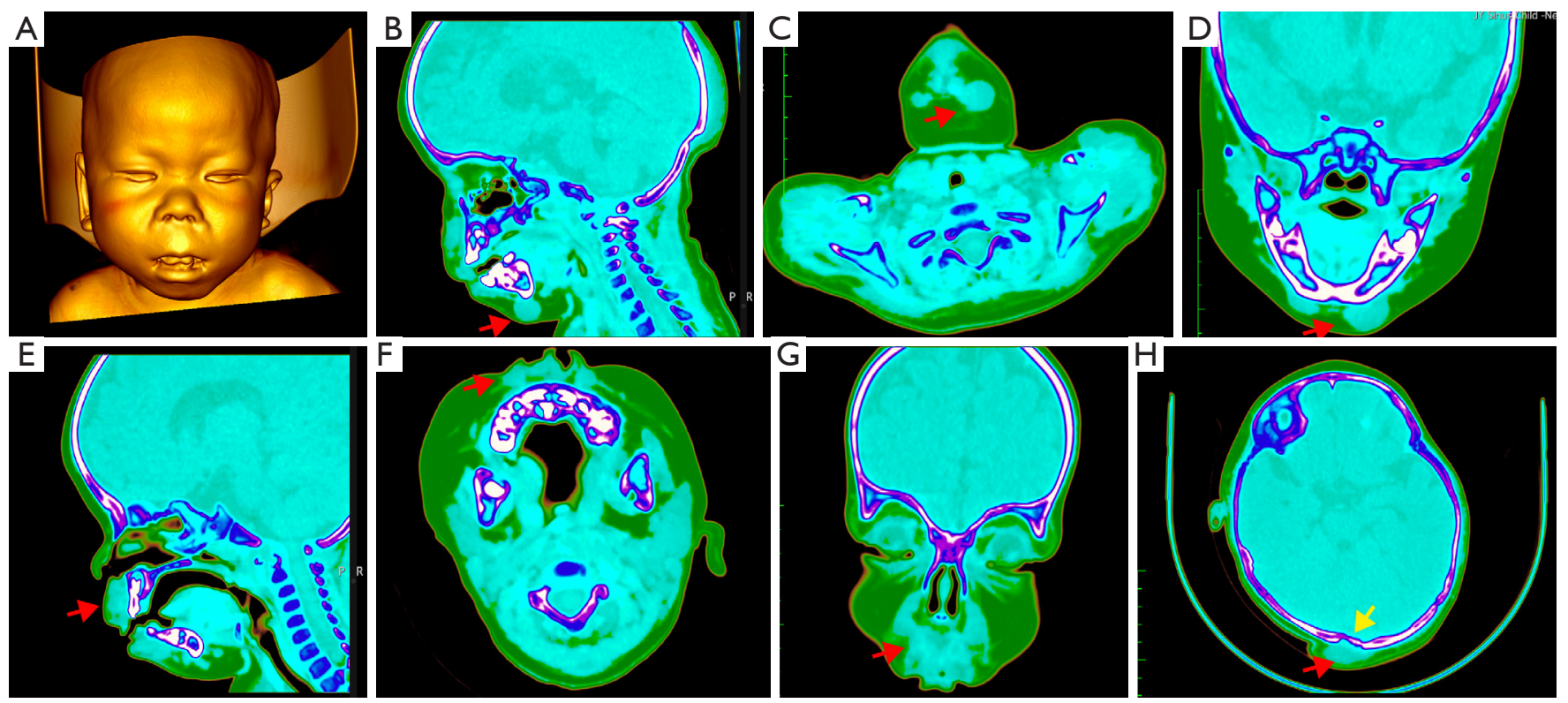

Figure 2 Maxillofacial CT scan of patient with JHF. (A) Three-dimensional CT reconstruction of the maxillofacial region; (B-D) sagittal, coronal and horizontal planes of the CT showed two chin masses connected with the periosteum closely (red arrows); (E-G) sagittal, coronal and horizontal planes of the CT showed swellings in the bilateral subnasal region (red arrow); (H) occipital mass (red arrow), occipital bone resorption defect (yellow arrow). These images are published with the consent from the patient's parent or legal guardian.
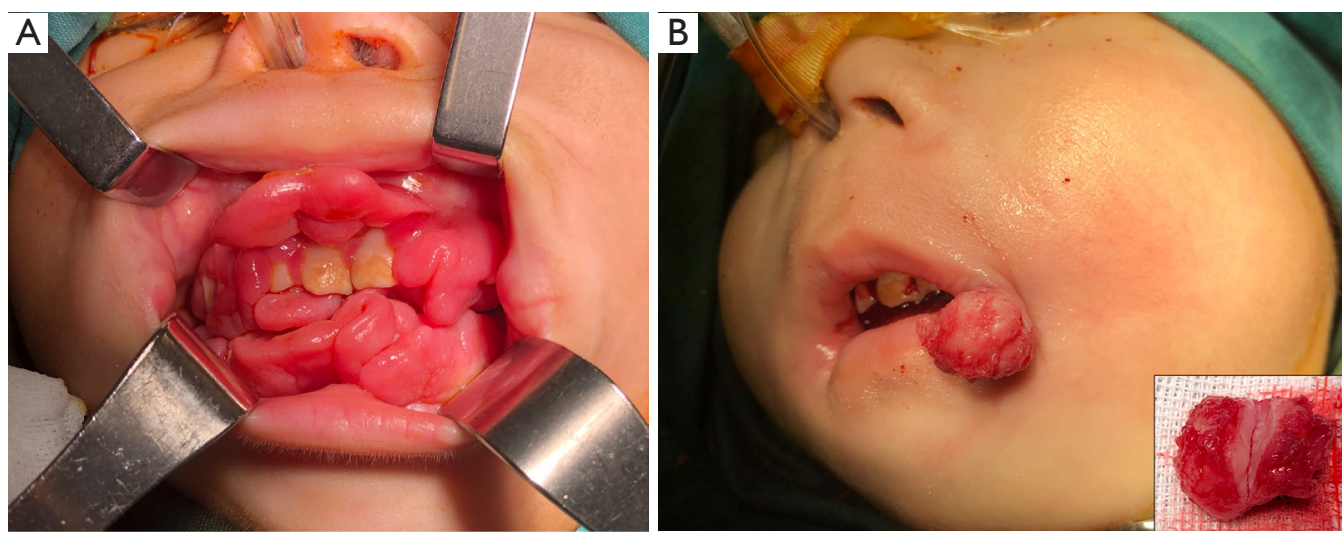

Figure 3 Intraoperative view. (A) Severe gingival hyperplasia covering the incisal margin of the teeth; (B) mass on the corner of the mouth, with no soft texture and a gray-white fiber-like cutting plane. These images are published with the consent from the patient's parent or legal guardian.

cells were positive for vimentin, but negative for smooth muscle actin (SMA) and S-100 protein (Figure 4D-4F).

In addition, the genome sequencing indicated a homozygous nucleotide mutation with the ANTXR2 gene. The homozygous variation in the ANTXR2 gene was inherited from the parents. Both parents were heterozygous, which was consistent with the autosomal negative inheritance mode.
Based on the clinical, pathological and genomic findings, the patient was diagnosed with "JHF".

All procedures performed in this study involving human participants were in accordance with the ethical standards of the institutional and/or national research committee(s) and with the Helsinki Declaration (as revised in 2013). Written informed consent was obtained from the patient's parent or legal guardian. 


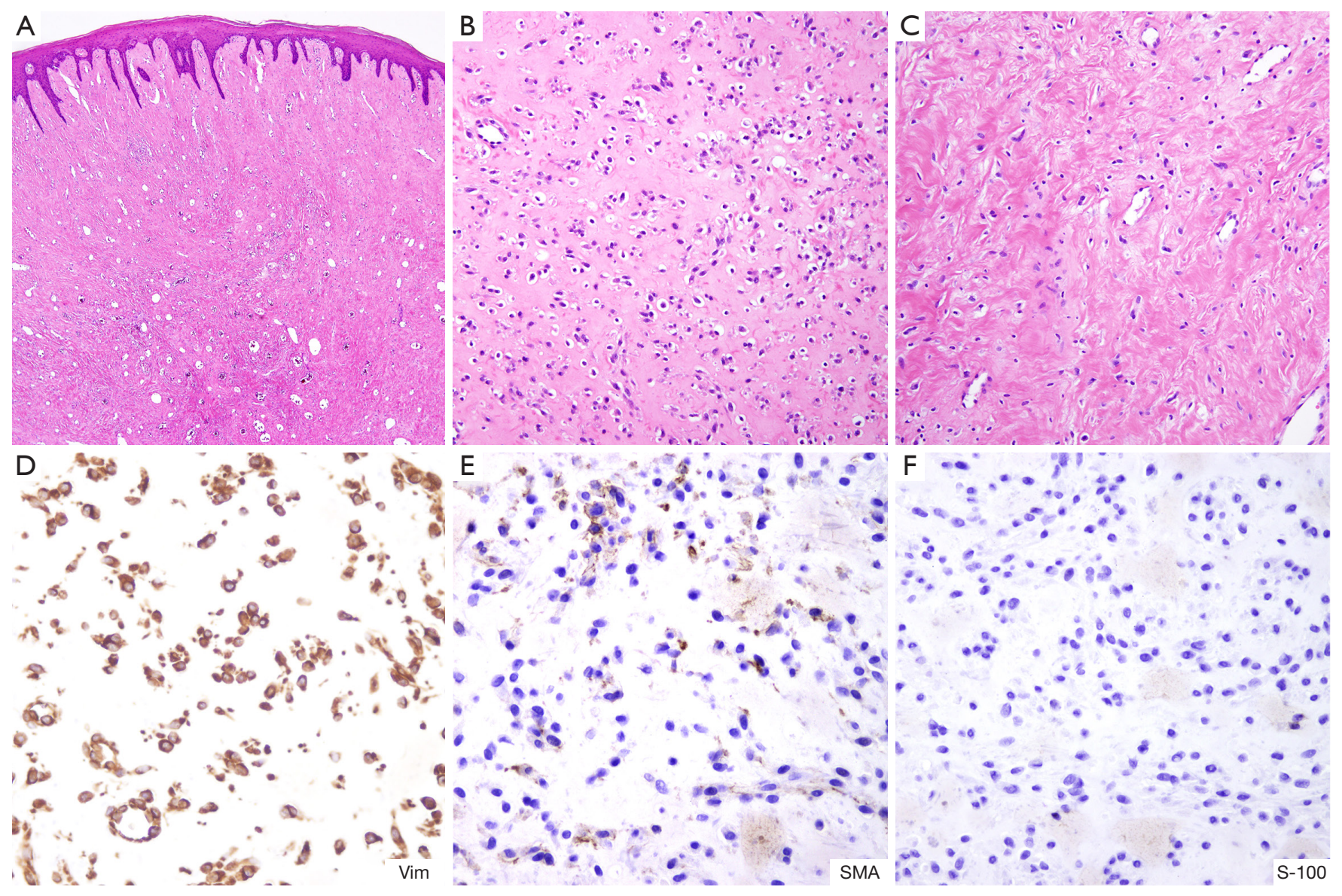

Figure 4 Biopsy micrograph. (A) The lesion is located in the submucosal layer, without obvious boundaries (H\&E staining, magnification 40x). (B) abundant monomorphic spindle-shaped cells proliferation accompanied with broadly homogeneous hyalinized matrix, indicating an early stage (H\&E staining, magnification 200x); (C) an advanced staged nodule showing a less cellular lesion with sparse single formed spindle-like cells surrounded by eosinophilic amorphous hyaline matrix (H\&E staining, magnification 200x); (D-F) positive for vimentin but negative for SMA and S-100 (IHC staining, magnification 400×).

\section{Discussion}

JHF is a very rare autosomal recessive genetic disorder that was described by Murray (6) in 1873 as "molluscum fibrosum" and then renamed by Kitano as JHF in 1976 (7).

Skin lesions are present at birth or develop in early childhood and usually vary widely in number and size. There may be small pearly papules or nodules, usually predominantly occurring on the head-and-neck region or on the face. Large subcutaneous tumors may also occur, particularly on the scalp. They may be hard or soft, fixed or mobile and they may ulcerate. Some skin lesions tend to recur following excision. Gingival hyperplasia is commonly present, interfering with dentition and feeding and leading to malnutrition. Patients with JHF present in childhood with joint contractures, osteopenia, and osteolytic bony lesions (8), as presented in this child patient. The musculature can be poorly developed. The condition persists into adult life, and the joint contractures are disabling.

It is genetically related to a similar, but not identical, condition termed infantile systemic hyalinosis (ISH) (9). It is essential to differentiate this condition from ISH which has a more widespread involvement of the viscera (diarrhea, recurrent infection, etc.) and a failure to thrive. Death usually occurs within 20 months of age (2). ISH, together with JHF, is considered to be a spectrum of hyaline fibromatosis syndrome (HFS) (10). The prognosis for these diseases is variable, with survival into adulthood for patients with JHF and early death from intractable diarrhea 
and recurrent infections in ISH. Both JHF and ISH are caused by genomic sequence variations in the ANTXR2/ $C M G 2$ gene, which encodes a protein involved in basement membrane matrix formation, in particular collagen type VI homeostasis, and morphogenesis of endothelial cells (11).

Histopathologically, skin lesions contain chondroitin cells embedded in an amorphous eosinophilic matrix. In early lesions, it is composed of glycosaminoglycans with abundant fibrous cells (as in Figure 4B), but in late lesions, the matrix is mainly composed of chondroitin sulfate with sparse spindled cells (as in Figure 4C) (12). In this case, gingival hyperplasia appeared earlier in the clinical stage and was at the late stage of the disease when surgery was performed, which showed a lesion with sparse cells surrounded by eosinophilic hyaline matrix, while submental masses appeared after gingival overgrowth, which was at the early stage of the disease, presenting a lesion with abundant cells and amorphous hyalinized stroma.

The treatment of JHF is generally symptomatic treatment and requires multidisciplinary care. Various therapeutic strategies have been used, including penicillamine, methotrexate, and steroids (both systemic and intralesional), unfortunately with limited success (13). Surgical excision is frequently performed for the treatment of subcutaneous nodules, and the recurrence rate is low. There were only several articles reporting the relapsed tumors $(2,14)$. Nodules may not respond to radiotherapy $(1,15)$. Gingivectomy is also common for the treatment of gingival hypertrophy. Joint contractures may respond to intralesional steroid injections in the early stages, as well as systemic steroid therapy. Karande and Andrade (8) reported that intralesional steroid therapy could also help control the symptoms of subcutaneous masses. Supportive care is an indispensable part of treatment. Pain is often managed with nonsteroidal anti-inflammatory drugs and even opiates (16). Physical rehabilitation has been advocated for a lifetime.

In addition, genetic counseling is an important aspect of management and needs to explain to parents an approximately $25 \%$ chance of recurrence in the next sibling (12). With the genetic defect being identified, prenatal diagnostic methods are likely to be invented in the near future.

\section{Conclusions}

JHF is a progressive disease characterized by subcutaneous nodules, gingival hyperplasia, joint contractures, and osteolytic lesions, that can cause functional and cosmetic impairment. There are no clear guidelines for the treatment. Early surgical treatment can be beneficial and results in sustainable outcomes. Supportive care is the mainstay of management.

\section{Acknowledgments}

Funding: This work was supported by the fundamental research program funding of Ninth People's Hospital affiliated to Shanghai Jiao Tong University School of Medicine (grant No. JYZZ114), and the National Key Research and Development Program of China (grant No. 2106YFC1000502).

\section{Footnote}

Reporting Checklist: The authors have completed the CARE reporting checklist. Available at https://dx.doi. org/10.21037/tp-21-169

Conflicts of Interest: All authors have completed the ICMJE uniform disclosure form (available at https://dx.doi. org/10.21037/tp-21-169). The authors have no conflicts of interest to declare.

Ethical Statement: The authors are accountable for all aspects of the work in ensuring that questions related to the accuracy or integrity of any part of the work are appropriately investigated and resolved. All procedures performed in this study involving human participants were in accordance with the ethical standards of the institutional and/or national research committee(s) and with the Helsinki Declaration (as revised in 2013). Written informed consent was obtained from the patient's parent or legal guardian for publication of this case report and accompanying images. A copy of the written consent is available for review by the editorial office of this journal.

Open Access Statement: This is an Open Access article distributed in accordance with the Creative Commons Attribution-NonCommercial-NoDerivs 4.0 International License (CC BY-NC-ND 4.0), which permits the noncommercial replication and distribution of the article with the strict proviso that no changes or edits are made and the original work is properly cited (including links to both the formal publication through the relevant DOI and the license). See: https://creativecommons.org/licenses/by-nc$\mathrm{nd} / 4.0 /$. 


\section{References}

1. Folpe AL, Schoen M, Kang S. Juvenile Hyaline Fibromatosis. Mayo Clin Proc 2020;95:328-9.

2. Casas-Alba D, Martínez-Monseny A, Pino-Ramírez RM, et al. Hyaline fibromatosis syndrome: Clinical update and phenotype-genotype correlations. Hum Mutat 2018;39:1752-63.

3. Çam B, Kurkcu M, Ozturan S, et al. Juvenile hyaline fibromatosis: a case report follow-up after 3 years and a review of the literature. Int J Dermatol 2015;54:217-21.

4. Marques SA, Stolf HO, Polizel JO, et al. Hyaline fibromatosis syndrome: cutaneous manifestations. An Bras Dermatol 2016;91:226-9.

5. Lyra ALCO, Razo LM, Estrella RR, et al. Juvenile hyaline fibromatosis: an unusual clinical presentation. Dermatol Online J 2019;25:13030/qt75082292.

6. Murray J. On three peculiar cases of Molluscum Fibrosum in Children in which one or more of the following conditions were observed: hypertrophy of the gums, enlargement of the ends of the fingers and toes, numerous connecive-tissue tumours on the scalp, \&c. Med Chir Trans 1873;56:235-254.1.

7. Kitano Y. Juvenile hyalin fibromatosis. Arch Dermatol 1976;112:86-8.

8. Karande V, Andrade NN. Juvenile Hyaline Fibromatosis. Contemp Clin Dent 2018;9:484-7.

9. Liu L, Ren F, Tan Q. Infantile Systemic Hyalinosis. JAMA Dermatol 2019;155:1306. Erratum in: JAMA

Cite this article as: $\mathrm{Xia} \mathrm{L}, \mathrm{Hu} \mathrm{Y}, \mathrm{Zhang} \mathrm{C}, \mathrm{Wu} \mathrm{D}$, Chen Y. Juvenile hyaline fibromatosis: a rare oral disease case report and literature review. Transl Pediatr 2021;10(11):3124-3129. doi: $10.21037 / \mathrm{tp}-21-169$
Dermatol. 2019 Nov 1;155(11):1323. doi: 10.1001/ jamadermatol.2019.3435.

10. Nofal A, Sanad M, Assaf M, et al. Juvenile hyaline fibromatosis and infantile systemic hyalinosis: a unifying term and a proposed grading system. J Am Acad Dermatol 2009;61:695-700.

11. El-Kamah GY, Fong K, El-Ruby M, et al. Spectrum of mutations in the ANTXR2 (CMG2) gene in infantile systemic hyalinosis and juvenile hyaline fibromatosis. Br J Dermatol 2010;163:213-5.

12. Ravikumar VR, Veerappan Ramamoorthi RG, Manisankar S. Juvenile hyaline fibromatosis in siblings. Indian J Pathol Microbiol 2019;62:300-2.

13. Al-Mayouf SM, AlMehaidib A, Bahabri S, et al. Infantile systemic hyalinosis: a fatal disorder commonly diagnosed among Arabs. Clin Exp Rheumatol 2005;23:717-20.

14. Urbina F, Sazunic I, Murray G. Infantile systemic hyalinosis or juvenile hyaline fibromatosis?. Pediatr Dermatol 2004;21:154-9.

15. Denadai R, Raposo-Amaral CE, Bertola D, et al. Identification of 2 novel ANTXR2 mutations in patients with hyaline fibromatosis syndrome and proposal of a modified grading system. Am J Med Genet A 2012;158A:732-42.

16. Shieh JT, Swidler P, Martignetti JA, et al. Systemic hyalinosis: a distinctive early childhood-onset disorder characterized by mutations in the anthrax toxin receptor 2 gene (ANTRX2). Pediatrics 2006;118:e1485-e1492. 